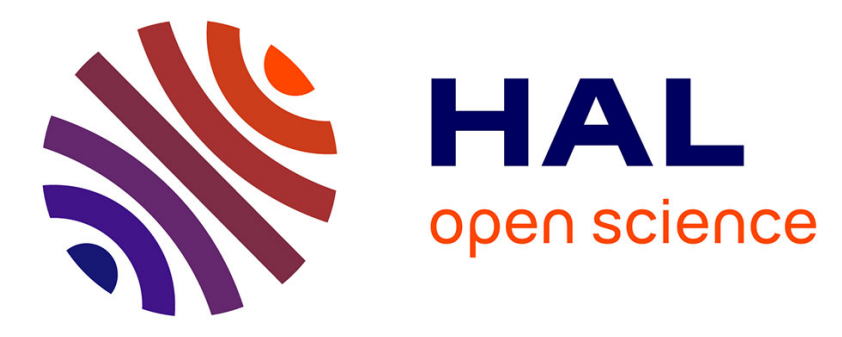

\title{
Analysis of the GPS acquisition environment : indoors and outdoors
}

\author{
Hanaa Al Bitar-El Natour, Anne-Christine Escher, Christophe Macabiau, \\ Marie-Laure Boucheret
}

\section{- To cite this version:}

Hanaa Al Bitar-El Natour, Anne-Christine Escher, Christophe Macabiau, Marie-Laure Boucheret. Analysis of the GPS acquisition environment: indoors and outdoors. ICTTA '06, 2nd IEEE International Conference on Information and Communication Technologies: from Theory to Applications, Apr 2006, Damascus, Syria. pp 127 - 132, 10.1109/ICTTA.2006.1684357 . hal-01021744

\section{HAL Id: hal-01021744 \\ https://hal-enac.archives-ouvertes.fr/hal-01021744}

Submitted on 27 Oct 2014

HAL is a multi-disciplinary open access archive for the deposit and dissemination of scientific research documents, whether they are published or not. The documents may come from teaching and research institutions in France or abroad, or from public or private research centers.
L'archive ouverte pluridisciplinaire HAL, est destinée au dépôt et à la diffusion de documents scientifiques de niveau recherche, publiés ou non, émanant des établissements d'enseignement et de recherche français ou étrangers, des laboratoires publics ou privés. 


\title{
Analysis of the GPS acquisition environment: indoors and outdoors
}

\author{
Hanaa E1 NATOUR Al BITAR ${ }^{1,2}$, Anne Christine ESCHER ${ }^{1}$, Christophe MACABIAU $^{1}$, Marie- \\ Laure BOUCHERET ${ }^{3}$ \\ $E N A C^{1}, \mathrm{TeSA}^{2}, \mathrm{ENST}^{3}$ \\ elnatour@recherche.enac.fr
}

\begin{abstract}
The emergence of the LBS services (Location Based Services) creates the need for indoor and outdoor positioning. Indoor environments refer to environments inside buildings, and outdoor environments refer to urban environments (streets). Indoor localization poses a true challenge for classical positioning systems. Indeed, radio propagation in indoor environments is affected by several factors particularly multipath, shadowing, cross-correlations, receiver mobility, interference... Urban environments are also challenging environments where multipaths problems are even more pronounced.
\end{abstract}

This paper aims at analyzing such environments in the frame of a state of the art of the GPS acquisition environment.

\section{Introduction}

The need for localization based on satellite constellation is dramatically increasing these days with the rising of Location Based Services (LBS) and other location and navigation applications, such as the E-911 and the E-112 mandates in the United States and Europe respectively. But the challenging indoor and outdoor environments cause big problems for this kind of positioning.

Globally, in order for GPS to work accurately, the presence of an unobstructed Line-Of-Site (LOS) signal is preferable. But such a LOS signal is not always available for GPS localization especially in indoor environments, where the obstructed signals become very weak thus creating cross-correlation problems, and in urban environments where multipaths are very likely to take place.

Cross-correlations are interferences caused by GPS signals on one another. This effect is particularly disturbing when the interfering signal is very strong compared to the searched signal. This is generally the case when one of the signals has reached the receiver through a window and the other passed through a wall or a ceiling for example.

Multipath consists of different replicas of the original signal caused by reflections, diffractions or diffusions generated when the signal encounters an obstacle. These replicas are attenuated versions of the original signal: they have different phases, and arrival times. At the receiver, they combine and produce a distorted version of the transmitted signal.
The GPS radio mobile environment is governed by many other factors like shadowing effects, surrounding objects velocity and mobile relative velocity. The shadowing effect mainly attenuates the direct signal. This attenuation is very severe when the signal must pass through concrete walls, or dense foliage. On the other hand, the mobile relative velocity with respect to the emitting satellite makes the channel variable over time.

In this paper, we mainly analyze the impact of crosscorrelation and multipath on GPS L1 signal acquisition in two different types of environments, namely the indoor and the urban environments and compare them. For that purpose, we have defined an indoor timevarying mobile radio channel model, based on an indoor channel model which was presented by the ESA (European Space agency) at ION-GPS-2003 [1]. In this paper, the ESA investigated a statistical non-timevarying model. The model we work with uses the distributions provided by the ESA to generate an original signal and many replicates. But, we assume the propagation delay to be time-varying and thus the carrier phase also. This model allows for studying the effect of either multipath, or GPs signal cross-correlations on the receiver output signal to noise density ratio $C / \mathrm{N}_{0}$ by the end of the acquisition process. To study urban multipath effects, we used another model also provided by the ESA and presented in the ION 2005. It is called "Land Mobile Multipath Channel" [2].

The paper will be organized as follows: first we introduce the general principles of the GPS system and the GPS receiver signal processing. The two subsequent parts show simulation results. The first part consists in presenting results corresponding to indoor environments, while the second deals with the outdoor environments.

\section{GPS general principles}

The GPS system uses a spread spectrum technique, based on CDMA modulation schemes, to ensure the channel multiple-access. The codes used for civil applications are Gold codes called C/A codes. Each satellite has its own C/A code. These codes have a period of 1023 chips. Their autocorrelation function is maximum for null a delay, and approximately equal to zero for non null delays. Their cross-correlation function is approximately equal to 0 for all delays. However, this is not always the case in practice as it will be shown next in the simulation results: the cross-correlation peaks level depends on the satellites respective power.

The GPS position determination is usually done by measuring the propagation delays of at least 4 GPS 
satellite signals. In order to measure the propagation delay of a particular signal, the receiver generates a replica of the studied signal code and carrier using its own clock. A classical receiver estimates the correlation function by multiplying the incoming signal with this replica at different time lags over a $1 \mathrm{~ms}$ interval (corresponding to one $\mathrm{C} / \mathrm{A}$ code period). If the code sample does not line up with the signal from the satellite, the sum of multiplied signal will be close to zero. The lags that are close to the correct time offset will sum to larger values (because more of the bits in the code line up). Once the satellite code and the replica code have both the same state, the receiver is able to determine its phase shift with respect to the received signal, and then deduce the propagation delay: this state corresponds to the maximum of the correlation function.

For each measure, the receiver can be positioned on a sphere centered on the signal emitting satellite and having as radius the calculated satellite-to-user range. The position can then be determined as the intersection of at least three spheres, as in Fig 1 . This is the principle of triangulation.
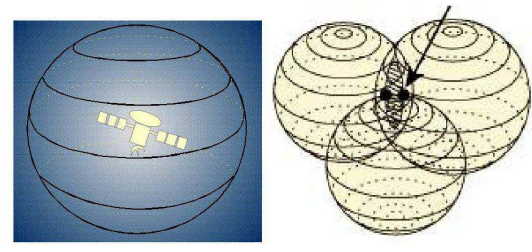

Fig 1: The triangulation method for determining the user position

But for accurate measurements, the GPS system must first proceed to the synchronization of the receiver with the transmitters to avoid the biases that may result from the signal propagation through the atmosphere and many other factors. In fact, the satellites are synchronized with each other since the GPS system provides a broadcast signal component that enables to determine the bias of each satellite clock with respect to a common clock, known as GPS time. So the only bias that affects the measurements is that between the receiver clock and the GPS time. This bias is an additional unknown to the three dimensional position unknowns. Consequently, 4 satellite signals are needed to compute an accurate $3 \mathrm{D}$ position.

The process of correlating the received signal with a generated C/A code replica is called despreading. It is achieved during the acquisition stage of the GPS signal processing. Acquisition is a coarse synchronization process giving estimates of the C/A code offset and the carrier Doppler for each satellite. The carrier Doppler is the result of the satellites and the receiver dynamics, and of the frequency drift of the receiver local oscillator.

This acquisition process is defined in an uncertainty region in which code and carrier phase are aligned with the received signal. The correct alignment is identified by the measurement of the output power. The result of this two dimensional search is an estimate of the code offset to within one half chip, and of the carrier Doppler to within half the Doppler search bin size (several hundred $\mathrm{Hz}$ ).

The final output is a 2-dimensional correlation matrix that contains a significant term (the useful signal) and two other noise terms that will eventually cause signal attenuation. One of these terms is an additive noise due to the signal propagation from the satellite to the user receiver, and the other noise term is due to the code delay and the carrier Doppler estimation errors. The next step is to find the right peak of the correlation matrix. The one which is likely to correspond to the right Doppler frequency and code delay of the GPS signal studied. As already explained, this detection matrix is maximal when the replica and the reference signal are aligned.

During our simulations, to insure that the Doppler and the code delay found at the end of the acquisition process are accurate, these two parameters are calculated $a$ priori for each signal. For a static receiver, the Doppler frequency is given by [3]:

$$
f_{d i}=L_{1} \cdot \frac{v_{d}}{c} \text {, where } L_{1} \text { is the civil GPS carrier }
$$
frequency, $v_{d}$ the considered satellite Doppler velocity and $c$ the velocity of light.

Next simulation results will be presented. The global acquisition time was not studied.

\section{Results for indoor environments}

To study the impact of different environments on the GPS acquisition, the sensitivity of the receiver will be studied. By sensitivity, we refer to the minimum $\mathrm{C} / \mathrm{N} 0$ that could be acquired for the same acquisition complexity.

\subsection{Multipath}

In order to study the impact of multipath on the acquisition performance, we will compare results obtained with a signal without multipath (i.e. just the LOS signal), another one containing a LOS and different multipath replicas, and a signal without a LOS, but only multipath replicas (Non-LOS). The signals used are generated using our channel model. Figure 2 shows the results for satellite number 1 . The Doppler frequency and the code delay for this signal are $F_{d}=-4666 \mathrm{~Hz}$ and $\tau=608.2758$ chips respectively.

This signal will be acquired also using the time-varying model we developed for this purpose.

The results below show the minimum $C / N_{0}$ value that could be acquired. The first value of the $C / N_{0}$ is that of the input $C / N_{0}$. The second one is the $C / N_{0}$ estimated by the acquisition algorithm at the end of the acquisition. Note that a loss of approximately $1.5 \mathrm{~dB}$ is due to the signal quantification process [4]. Recall that the signal is said to be acquired if the acquisition output matches with the right values of the code delay and the Doppler frequency of the considered satellite. 
As it is illustrated in Fig 2 below, for a LOS signal without multipath replicas the minimum $C / N_{0}$ that could be acquired is $21 \mathrm{dBHz}$.

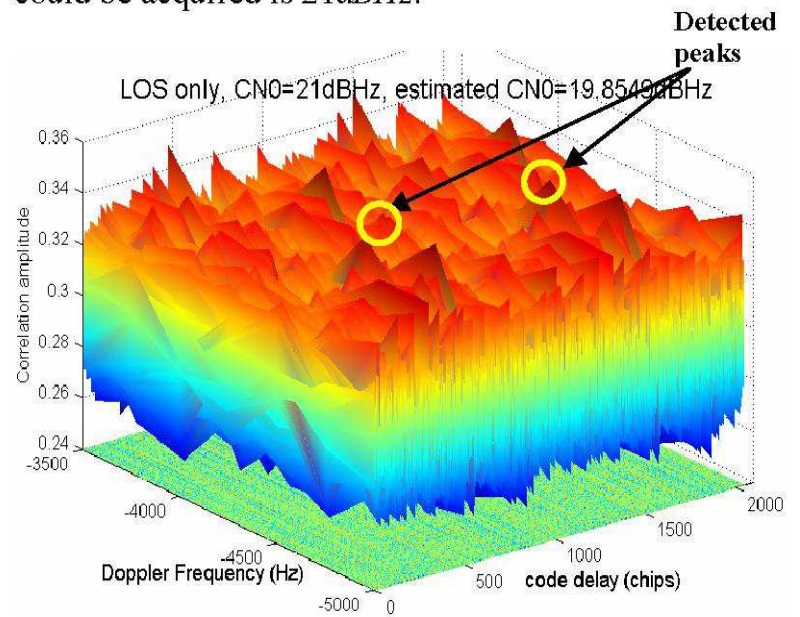

Fig 2: LOS without multipath replicas; initial $C / N_{0}$ 21dBHz; estimated $C / N_{0} \quad 19.8549 d B H z$

In the presence of a LOS with multipath replicas, the minimal $C / N_{0}$ that could be detected in this case was also $21 \mathrm{dBHz}$ (Fig 4).

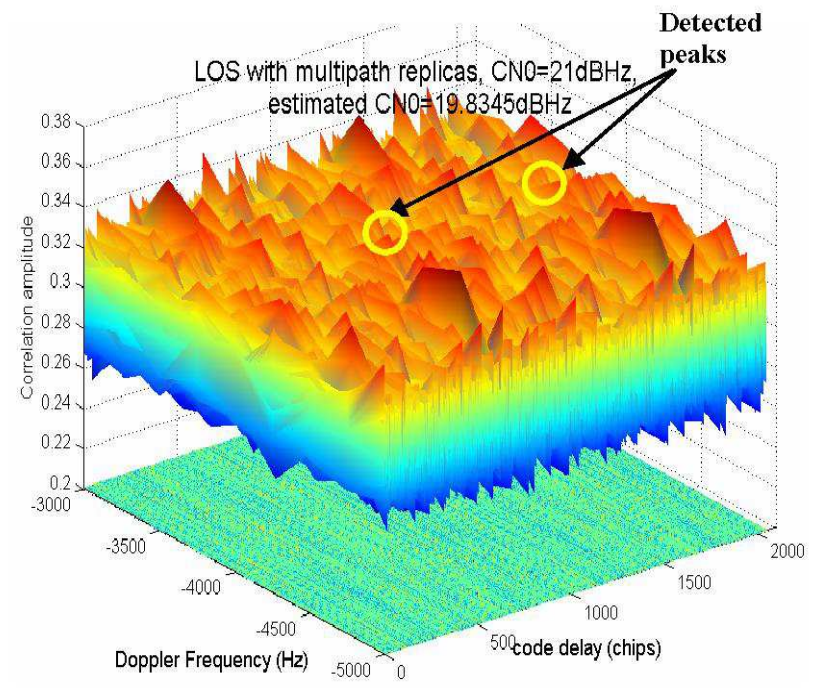

Fig 3: LOS signal with multipath replicas; initial $C / N_{0}$ 21 $\mathrm{dBHz}$; estimated $C / N_{0} \quad 19.8345 \mathrm{dBHz}$

We notice that the estimated $C / N_{0}$ in these two cases is very close to the real one, and the values of the Doppler and the code delay are accurate. Hence multipath replicas did not disturb the acquisition process.

As depicted in Fig 4, in the case of a Non-LOS composite signal, the minimum $C / N_{0}$ ratio that could be detected is rather $40 \mathrm{dBHz}$. In other words, if a direct LOS signal had a $C / N_{0}$ ratio lower than $40 \mathrm{dBHz}$ and could not reach the receiver (because of shadowing, for instance), the multipath replicas generated would not allow for finding the desired signal.

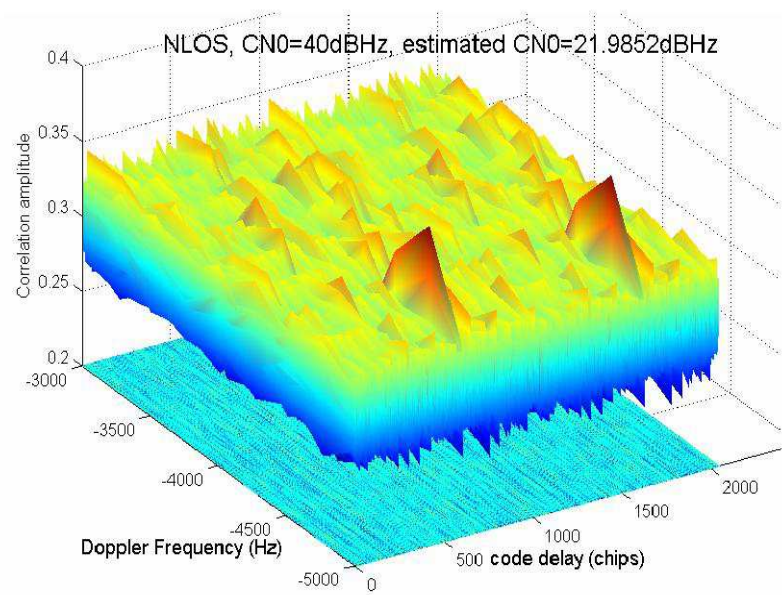

Fig 4: Non-LOS signal, multipath replicas only; initial $C / N_{0} 40 \mathrm{dBHz}\left(C / N_{0}\right.$ that affected the LOS that could not reach the receiver); estimated $C / N_{0} 21.9852 \mathrm{dBHz}$

In this case we found that the multipath replicas were severely attenuated with respect to the LOS.

These tests were repeated for many different signals, and the results obtained were similar to those presented here when a LOS reaches the receiver.

As a conclusion, the multipath replicas have minor impact on the acquisition performance, when a direct LOS is present. In addition, even if a LOS is not available, the multipath replicas could be explored, but they are much attenuated with respect to the LOS signal. In all cases multipath replicas did not induce errors on the signal acquisition. On the other hand, in Non-LOS cases, the minimum $\mathrm{C} / \mathrm{N} 0$ of the direct (masked) signal for successful acquisition depended on the indoor environment considered (25-40 $\mathrm{dBHz}$ ).

\subsection{Cross-correlations}

After having studied the effect of multipath on signal fading in indoor environments, the next step will be to study the impact of interference between different satellite signals. In fact there are situations where a strong path may interfere with another weak path. In this case, acquiring the weak signal is very difficult, and may lead to the detection a cross-correlation peak. This crosscorrelation peak is expected approximately $-23.9 \mathrm{dBHz}$ apart from the autocorrelation peak [5]. Thus theoretically, if 2 signals have $\mathrm{C} / \mathrm{N} 0$ ratios about $24 \mathrm{dBs}$ apart from each other, the autocorrelation peak of the weaker signal is approximately at the same level as that of the strong signal cross-correlation peaks. To illustrate this situation, we used the same model that was developed for the indoor multipath simulations, and extended it to support different satellite signals. We tried two satellite signals interfering with each other, with different powers. Obviously, corss-correlations are particularly disturbing in the case of 2 signals with one of them being too strong (a direct LOS for example) and the other too weak (due to shadowing for example). The results of 2 GPS signals (for satellites number 1 and 11) and their multipath versions are presented. The $C / N_{0}$ 
ratio is set to $50 \mathrm{dBHz}$ for the first one and $25 \mathrm{dBHz}$ for the other one. The 2 satellites have different code delays and Doppler frequencies. We tried to acquire the satellite number 11. The result of acquisition is depicted in Fig 5 .

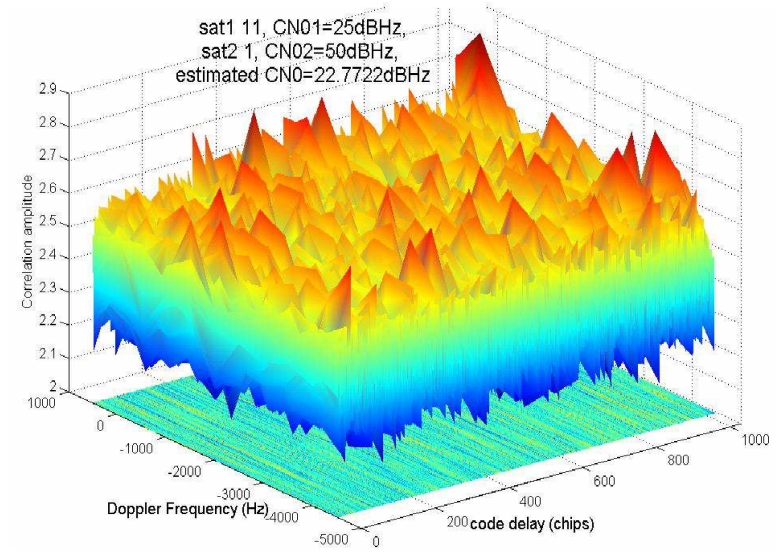

Fig 5: Cross-correlation between 2 GPS signals: 1- signal of satellite $11, C / N_{0} 25 \mathrm{dBHz}$ 2- signal of satellite $1, C / N_{0}$ $50 \mathrm{dBHz}$. The signal acquired is that of sat 11. Estimated $C / N_{0} 22.7722 d B H z$

The peak detected after acquisition does not match with the code delay and Doppler frequency of satellite 11. In addition, this peak is found at approximately $-27 \mathrm{dBHz}$ less than the initial $C / N_{0}$ applied to the signal of this satellite, namely $50 \mathrm{dBHz}$. By adding the attenuation due to the quantification, the estimated $C / N_{0}$ corresponding to this peak will be very close to the theoretical value, $-25 \mathrm{dBHz}$ approximately. This leads us to assume that this peak is very likely to be a cross-correlation one, not a noise peak.

In order to be sure that it is a cross-correlation peak, we acquired the signal of satellite 1 alone without noise, but used the C/A code of satellite 11 in this acquisition. The detected peak has the same (Doppler frequency, code delay) couple as that of figure 5 .

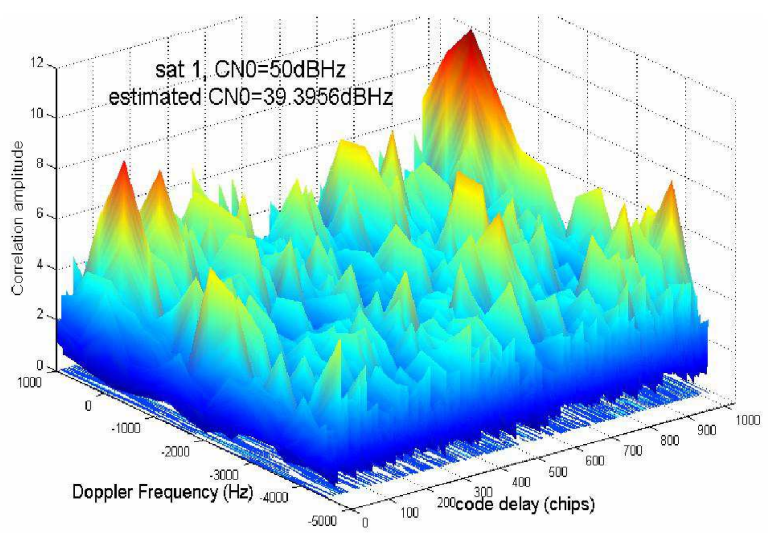

Fig 6: LOS signal of satellite 1 correlated with C/A code of satellite 11 to view the resulting cross-correlation function.

Generally, the cross-correlation matrix is not supposed to have peaks which are stronger than others. Theoretically, all peaks must have the same amplitude.
But this is not the case in Fig 6 where many peaks are stronger than others. This may be due to the effect of the code Doppler drift which may cancel some crosscorrelation peaks.

Figures 5 and 6 show that the cross-correlation disturbs the acquisition of a weak signal in the presence of a strong one. The weakest signal namely that of satellite 11 at $25 \mathrm{dBHz}$ could be easily acquired if there were not an interfering signal.

In conclusion, the main impact of GPS crosscorrelation on the acquisition performance is a probable cross-correlation peak, which leads to inaccurate values of the Doppler frequency and the code delay, and hence to a bad estimation of the user position estimation.

\section{Results for outdoor environments}

\subsection{Mutlipath}

To study outdoor multipath we will use a model developed by the ESA for this purpose. This model is based on both deterministic and stochastic processes within an artificial scenery that can be parameterised. Figure 7 below presents an example of an urban environment simulated using this model [2].

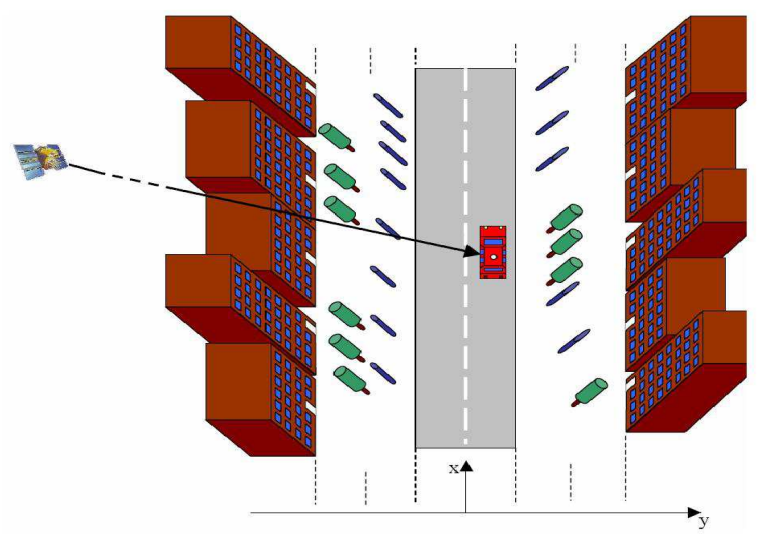

Fig 7: Artificial scenery [2]

For this model, the GPS receiver is assumed to be onboard a car that is moving in the north direction. All the components of this scenery can be parameterized, like the car velocity, the road width, the building and house heights and shapes, the trees and pole positions, the satellite position, etc. For the sake of simplicity, the buildings shape is considered to be constant, only the impact of the satellite position and the road width will be studied. In this paper, the satellite position is determined by its azimuth and elevation angles. The result is a Probability Density Function (PDF) with respect to time and signal power.

First an elevation angle of $60^{\circ}$ and a road width of $4 \mathrm{~m}$ will be considered. Three different cases are tested according to the value of the azimuth angle: (1) $0^{\circ}$ (the satellite is in the direction of the car movement), (2) $30^{\circ}$, (3) $60^{\circ}$. Figures 8 to 10 depict the PDF as a function of time and signal power for each of these cases. 


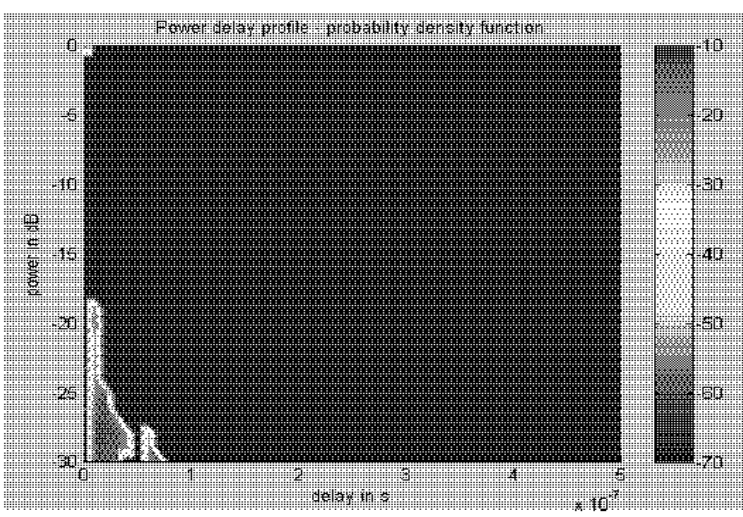

Fig 8: Power Delay Profile (PDP) and PDF for a satellite elevation angle of $60^{\circ}$, an azimuth of $0^{\circ}$ and a road width of $4 \mathrm{~m}$

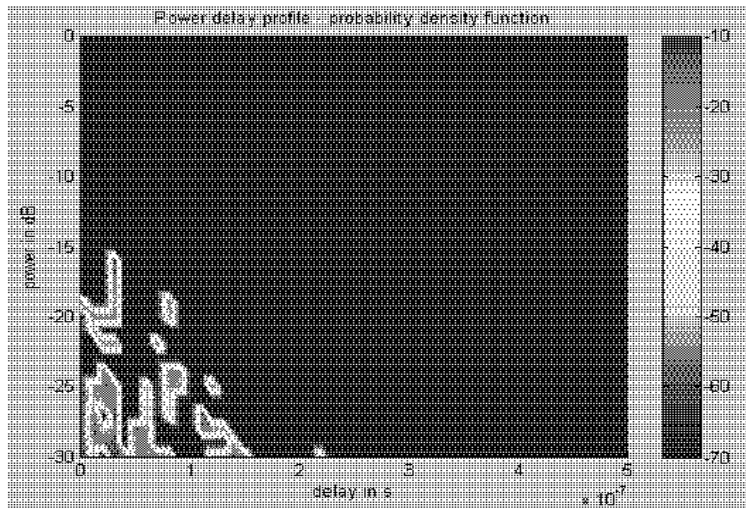

Fig 9: (PDP) and PDF for a satellite elevation angle of $60^{\circ}$, an azimuth of $30^{\circ}$ and a road width of $4 \mathrm{~m}$

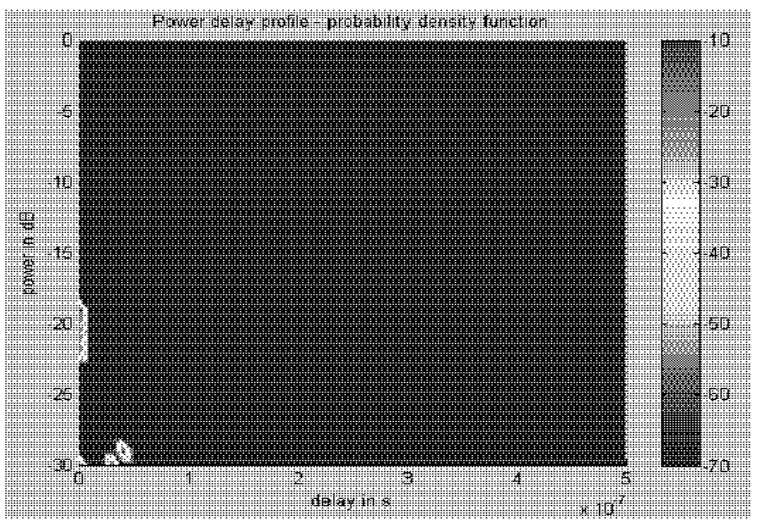

Fig 10: (PDP) and PDF for a satellite elevation angle of $60^{\circ}$, an azimuth of $60^{\circ}$ and a road width of $4 \mathrm{~m}$

The best performance is obtained in the first case where an obstructed LOS could reach the receiver in parallel with many multipath replicas (The multipath replicas have delays greater than $0 \mathrm{~s}$ ). But in this case, the multipaths are nut disturbing because they are all much weaker than the LOS. Hence the user position can be determined accurately. In the second case, where the satellite azimuth is set to $30^{\circ}$, the multipath replicas are stronger than the LOS signal and thus lead to false detection (detection of a wrong peak). Note that the replicas which are stronger than the LOS signal have an approximate delay of $3 \cdot 10^{-2}$ and $9 \cdot 10^{-2} \mu \mathrm{s}$. Such an error on the code delay will result in a position error of approximately $9 \mathrm{~m}$ and $18 \mathrm{~m}$ respectively in the horizontal plane. This could be predicted since the respective channel impulse responses obtained for the cases of figures 8 and 9 show clearly the attenuation of the LOS for an azimuth of $30^{\circ}$

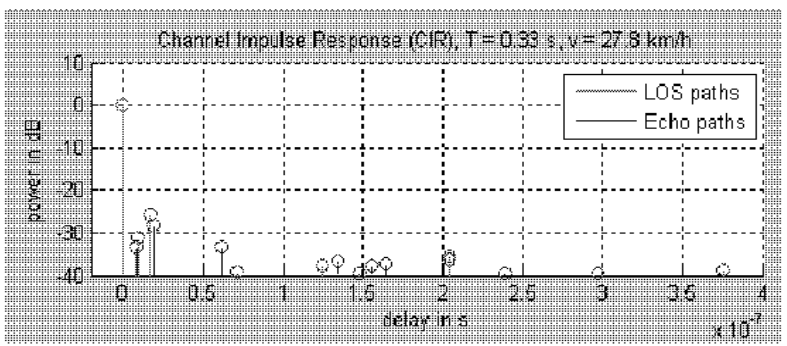

Fig 11: Channel Impulse response corresponding to figure 8

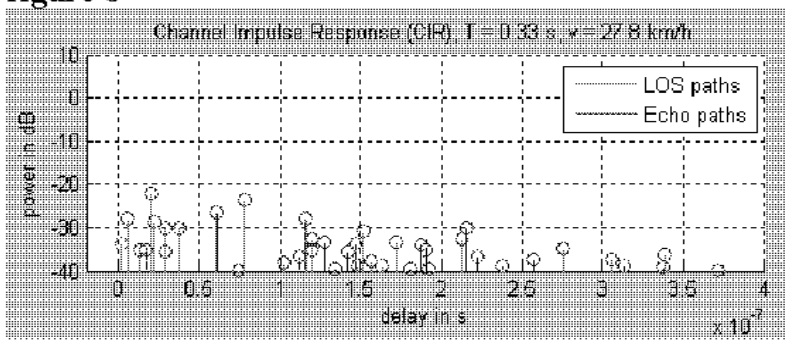

Fig 12: Channel Impulse response corresponding to figure 9

In the last case (azimuth of $60^{\circ}$ ), we expected that the performances would be worse than for the 2 previous cases $\left(0^{\circ}\right.$ and $\left.30^{\circ}\right)$. But this was not really the case as it can be seen in figure 10 . This is due to the considered environment and the separation between the buildings which is more favorable for a greater azimuth. The signal fading noticed in figures 9 and 10 is due to crossing eventual buildings roofs or walls.

A road width of $4 \mathrm{~m}$ corresponds to narrow streets. A large road $(30 \mathrm{~m})$ is considered in the next figure (with trees on its borders as it is generally the case).

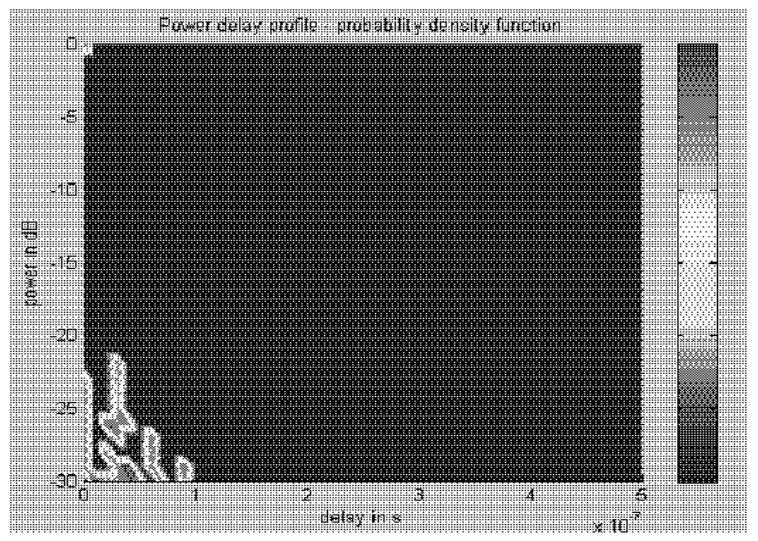

Fig 13: (PDP) and PDF for a satellite elevation angle of $60^{\circ}$, an azimuth of $30^{\circ}$ and a road width of $3 \mathrm{~m}$

The comparison between figures 11 and 8 shows that in both cases an unobstructed LOS signal could reach the 
receiver, but in figure 11 additional attenuated LOS signals, that may have crossed tress or building walls, reached the receiver because the environment is wider.

In conclusion, urban multipath is particularly disturbing in confined environments such as down towns, or small towns. The simulations conducted show that as for of indoor multipath, when a sufficiently strong LOS signal is present, the multipath is not disturbing. But for outdoor environments, the LOS is more likely to be resent than in indoor environments, where even if it reaches the receiver, it will be attenuated.

\subsection{Cross-correlations}

Cross-correlations were also studied for urban case using our model first. The results obtained for confined environments were similar to those obtained for indoor cases. This is because in such environments a satellite at low elevation angle for example is very likely to be attenuated due to shadowing effects (trees, building walls, roofs,...). In order to highlight this situation, the ESA model was used setting the azimuth and the elevation angles to $60^{\circ}$ and $30^{\circ}$ respectively. The road width is set to $4 \mathrm{~m}$. The result is depicted in figure 12 .

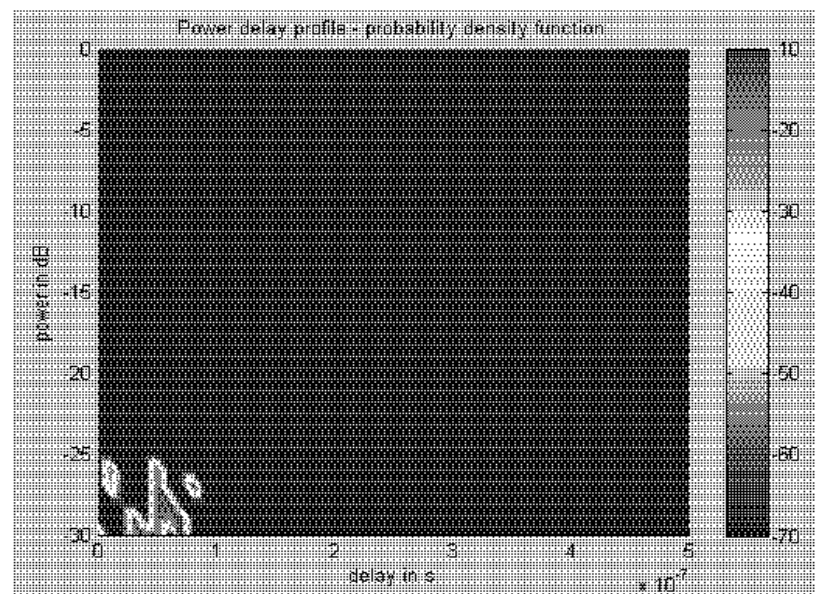

Fig 14: (PDP) and PDF for a satellite elevation angle of $30^{\circ}$, an azimuth of $60^{\circ}$ and a road width of $4 \mathrm{~m}$

According to figures 14 and 8 it can be concluded that in such urban environments a large power discrepancy may exist between 2 satellite signals reaching the receiver together. This is a typical situation leading to cross-correlation problems. Such problems are uncommon in wide urban environments. Indeed it is also the case for indoor environments, where the signals are all weak signals, and a gap of $25 \mathrm{dBHz}$ between them is not very common.

\section{Conclusion}

In this paper we investigated the impact of Multipath replicas and cross-correlation on the GPS signals acquisition for indoor and outdoor environments.
Indoor environments were studied using a time varying model developed for his purpose, while outdoor environments were rather studied using a model developed by the ESA. Three types of indoor GPS signals were generated and tested: a direct LOS signal, a LOS signal with multipath replicas, and a Non-LOS signal which contains only multipath replicas. The crosscorrelation effect was studied by adding two of these signals together.

We focused our work on the capacity of detection of a correlation peak or the estimation of the received signal power, and we did not analyze the total time needed for acquisition.

The results show that for indoor cases the multipath replicas do not disturb the acquisition performance, when a direct LOS is present. And even if a NLOS case they could be explored, but they are much attenuated compared to the LOS signal. However, the presence of the a direct LOS in such environments is not always possible, and even if a LOS reaches the receiver, it is generally too attenuated. Cross-correlations are particularly disturbing when a strong and a weak signal reach the receiver together. But this is not very common unless a signal reaches the receiver through a window for example.

Where as in urban environments, multipaths are not very disturbing unless the LOS is attenuated (due to trees or building walls). This is particularly the case for confined urban environments. But it is common to receive two or more satellite signals with power levels very apart from each other, and cross-correlations are more likely to occur.

\section{References}

[1] F. Pérez-Fontán, B. Sanmartin, A. Steingass, A. Lehner, J. Selva, E. Kubista, B. Arbesser-Ratsburg, "Measurements and Modeling of the Satellite-to-indoor Cahnnel for Galileo", Proceedings of the ION-GPS2003, Oregon Convention Center, Portland, Oregon, September 9-12, 2003.

[2] A. Steingass, A. Lehner (2005), "A Channel Model for Land Mobile Satellite Navigation", GNSS 2005 Conference, Munich, Germany, July 19-22, 2005.

[3] Eliott D. Kaplan, "Understanding GPS: Principles and Applications", Artech House, Norwood, MA, 1996

[4] J.J Spilker Jr. and B. W. Parkinson, "Global Positioning System: Theory and Applications Volume I, American Institute of Aeronautics and Astronautics", Inc, Washington DC, 1996.

[5] J. G. Proakis, "Digital Communications", Third Edition, McGraw-Hill, 1995. 